\title{
A REVIEW ON WIRELESS POWER TRANSFER: CONCEPTS, IMPLEMENTATIONS, CHALLENGES, AND MITIGATION SCHEMES
}

\author{
M. B. Sidiku',*, E. M. Eronu ${ }^{2}$ and E. C. Ashigwuike ${ }^{3}$

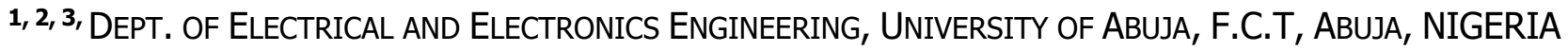 \\ Email addresses: ${ }^{1}$ smosunmola@gmail.com, ${ }^{2}$ eronu.majiyebo@uniabuja.edu.ng, \\ 3 ecashigwuike@ymail.com
}

\begin{abstract}
This paper reviews the current strides in the wireless power transfer (WPT) system. The paper discusses the classification of wireless power transfer, its application, trend and impact on society, advantages as well as disadvantages. It also presents a comparative analysis of existing work done by researchers in the field of wireless power transfer showing the shortcomings in various topologies, communication, and optimization methods used to increase the overall performance efficiency and proffer direction for further studies.
\end{abstract}

Keywords: wireless power transfer, application, advantages, disadvantages topologies, communication, optimization, efficiency

\section{INTRODUCTION}

For a very long time, electricity transfer has largely been through the use of conventional wire system. The traditional cable (wire) power transmission has the problem of aging, wear, and produces electric spark. It is difficult to use the cable to transmit in certain terrains such as high mountains and sea beds due to the negative impact this has on the safety and reliability of its use [1]. Wireless Power Transfer (WPT) can cause a paradigm shift by completely revolutionizing the mode of transmitting power. WPT is an enormous strategy for transmitting electric power from one point to the other through a vacuum or atmosphere over a distance without the usage of traditional cables or any other substance [2].

Wireless charging has evolved from mere theoretical concepts towards standard features on commercial products, especially mobile phones and portable smart devices [3]. The year 2014 witnessed the production of contemporary high-end smartphones by mobile giants such as Huawei and Samsung. These devices were equipped with wireless charging functionality. Currently, several companies, like Qualcomm, Evatran, WiTricity, etc have already developed products capable of transferring power with acceptable efficiency through a $15-30 \mathrm{~cm}$ air gap. This paper presents a general overview of the wireless power transfer scheme.

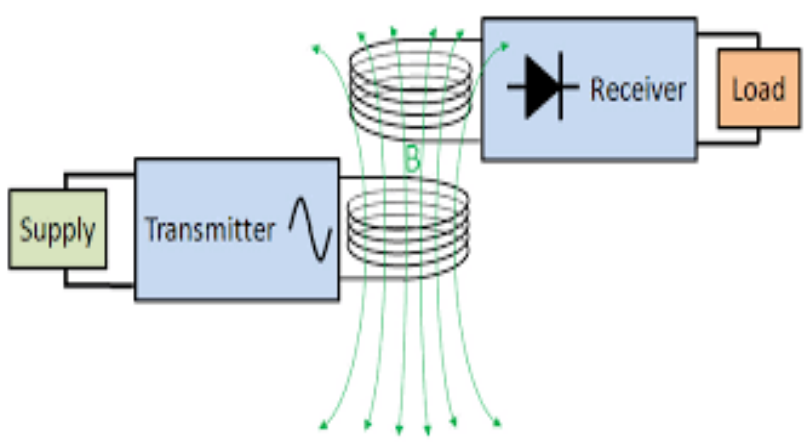

Figure 1: Wireless Power Transfer

\section{THEORETICAL BACKGROUND}

According to the energy transfer mechanism [1], WPT can be categorized into two types, namely: Farfield and Near field WPT system [2].

\subsection{Far-Field Wireless Power Transfer}

Far-field wireless power transfer which is also called electromagnetic radiation WPT[1], adopts electromagnetic waves like radio frequency signals as a medium to deliver energy in a form of radiation[3]. This is then transferred by the electric field of an electromagnetic wave, which is radiative

\footnotetext{
* Corresponding author, tel: +234-808-492 - 2145
} 
[3]. Far-field WPT includes microwave power transfer (MPT), Laser Power Transfer (LPT), and Solar Power Transfer (SPT) [2].

\subsubsection{Microwave Power Transfer (MPT)}

Microwave Power Transfer (MPT), which is based on electromagnetic radiation, utilizes the far-field radiation effect of the electromagnetic field to transfer power in free space. [4]. A high power transmission level is ensured using this technology when initialized at the base stations and fed to the mobile devices and receiving station. For this to be effective, two points must fall within the line of sight. With the aid of Magnetron, the technology, when deployed with geosynchronous transmission and reception satellites, boosts the power of objects obtained from the base station.

MPT is effective in the area of energy conversion; however, the difficulty experienced in trying to focus the beam over a small area presents challenges [5]. Power transmission begins with the conversion of electrical energy to microwaves, which is then captured by the rectenna. In using this technology, Alternating Current (AC) is not directly converted to the required microwave energy. Conversion to Direct Current (DC) must first be done and then to microwave utilizing magnetron. The rectenna receives the transmitted waves and efficiently changes the microwaves to electricity in the form of $\mathrm{DC}$, then back to AC [5]

\subsubsection{Laser Power Transfer (LPT)}

Laser power transfer (LPT) transmits power under visible or near-infrared frequency. It uses highly concentrated laser light aiming at the energy receiver to achieve efficient power delivery over long distances. The receiver of laser powering uses specialized photovoltaic cells to convert the received laser light into electricity [6]. LPT has an advantage of energy concentration. However, laser radiation could be hazardous and it requires Line of Sight (LOS) link as well as accurate pointing towards the receiver which could be challenging to achieve in practice [7]. It also requires complicated tracking mechanisms and a large spectrum of devices. Compared to microwave WPT, laser beaming is more vulnerable to atmospheric absorption and scattering by clouds, fog, and rain, which greatly hinders its practical applications [8]. A strong laser beam constitutes serious health hazards to humans and this method is quite expensive to actualize [9].

\subsubsection{Solar Powered Satellite (SPS)}

It is the largest application of WPT and it makes use of satellites with giant solar arrays and placing them in Geosynchronous Earth Orbit. These satellites play a pivotal role in generating and transmitting power as microwaves to the earth [10].

\subsection{Near Field Wireless Power Transfer}

Near field WPT which is also known as non-radiative wireless charging is based on the coupling of the magnetic field between two coils within the distance of the dimension of the coil for energy transmission [11]. As the magnetic field of an electromagnetic wave attenuates much faster than the electric field, the power transfer over a distance is largely limited. As a result of its safety merits, everyday home appliances as trivial as toothbrushes to more sophisticated machines like electric vehicles have been designed using non-radiative wireless charging. The Nearfield WPT can be classified into two groups namely: Inductive Power Transfer (IPT) and Capacitive Power Transfer (CPT). The inductive power transfer can further be divided into Inductive Coupled Wireless Power Transfer (ICWPT) and Magnetically Coupled Resonance Wireless Power Transfer (MCR WPT) [1]. Although the aforementioned methods vary in transferred distance, efficiency, frequency, transferred power, and resonator dimensions, they all work to achieve wireless power transfer needed for charging. Their workings contrasted as follows [12].

\subsubsection{Inductive Coupled Wireless Power Transfer}

The Inductive coupled WPT is the most used method for wirelessly charging low powered devices so far [13]. It transfers power from one coil to another and has been used for powering RFID tags, medical implants [4], in the fields of sensors, wirelessly charging electronic devices and in the car manufacturing industry. The operating frequency of inductive coupling is in the kilohertz range and is typically used within a few millimeters to a few centimeters $(20 \mathrm{~cm})$ from the targeted load [4] and its power varies between watt and kilowatt based on transmission efficiency [4].

The advantages of the inductive coupling WPT system include ease of implementation, convenient operation [14]. It is non-radiative and due to its low transmission frequency, it is considered safe for humans $[2,4]$. It has a high transfer efficiency of up 
to $95 \%$ at short distances [2], it eliminates sparks and other hazards in situations like coal mining and there is no danger of either electrocution or short circuit under any power range condition as a result of the coupling being magnetic [12]. However, a limitation of standard inductive charging is that it performs well in considerably short distances of communication, increasing the communication distance adversely drops the performance.

\subsubsection{Magnetically Coupled Resonance Wireless Power Transfer (MCR WPT)}

Magnetically coupled resonance wireless power transfer (MCR-WPT) follows the same basic principles as Inductive coupled wireless power transfer. It also transmits power from a source to a load [15]. However, this magnetically coupled resonant makes use of magnetic resonant coils, which operate at the same resonance frequency [4]. By using magnetically coupled resonant over standard inductive coupling, it is possible to achieve up to fifty percent efficiency for power transmission over longer distances without uttering coil size and power consumption [16]. MCR-WPT technology has attracted significant attention from academia and industries because it can cover all aspects of human life from consumer and medical electronics, smart homes to electric vehicles, showing great potential for further application.

The operating frequency ranges from a few hundred $\mathrm{kHz}$ and tens of MHz. MCR WPT has advantages of long-distance within several meters, unaffected by weather environments [2], line of sight (LOS) is not required when devices are charged, compared to Inductive coupled wireless power transfer, Magnetically Coupled Resonance Wireless Power Transfer has higher transfer power and efficiency and is considered to be one of the most potent techniques for mid-range WPT applications at present [17]. The non-radiative nature of the Coupled magnetic resonant system presents no threat to the environment when compared with the microwave and laser WPT. However, MCR WPT experiences a decrease in efficiency as a result of the axial mismatch between the receiver and transmitter coils, decreased efficiency with increased distance, and complex implementation [2].

\subsubsection{Capacitive Power Transfer (CPT).}

Capacitive Power Transfer (CPT) involves the transmission of energy between electrodes such as metal plates. A charged retaining capacitor is formed by receiver and transmitter electrodes. The transmitter creates an alternating voltage on the transmitting plate, from which the oscillating electric field via electrostatic induction induces on the receiver plate, an alternating potential, which turns into alternating current flow in the load circuit. [18]. Though capacitive power transfer is cheaper than Inductive coupling and magnetically coupled resonant, however, CPT requires close contact between the two metal surfaces. Hence, it is greatly limited by range requirements[4] The major drawback with capacitive WPT systems is that electric fields do not share the safety characteristics of magnetic fields, since their relative field strength is much greater, posing a hazard to both humans and electronic devices $[4,5]$.

Also, the achievable amount of coupling capacitance is dependent on the available area of the device. However, it is difficult to create sufficient power density required for charging when considering normal-sized portable electronic devices which indeed poses a design challenge [11].

\section{WIRELESS POWER TRANSFER DEVELOPMENT TRENDS AND IMPACT ON SOCIETY 3.1 Rezence by the Alliance for Wireless Power (A4WP)}

Rezence is a magnetic resonance wireless power transfer standard developed by the Alliance for Wireless Power (A4WP). A single transmitter can supply power to up to eight receiver devices on midrange distances. Communication between transmitters and receivers is "out-of-band" and implemented via Bluetooth. The A4WP was founded in early 2012 to develop a ubiquitous WPT ecosystem. The members of PMA are drawn from every known stratum of the telecommunication industries such as Broadcom, Panasonic, Microsoft Corporation, LG Electronics, Samsung, Logitech, WiTricity, Qualcomm, Incorporated, Gill Electronics, Hewlett Packard, Integrated Device Technology, Inc., Intel and other [25].

\subsection{Qi Technology}

The Qi (pronounce "Chee") was the first introduced wireless charging standard in the year of 2008 [19]. Qi Wireless PowerTransfer standard works on the principle of magnetic induction 


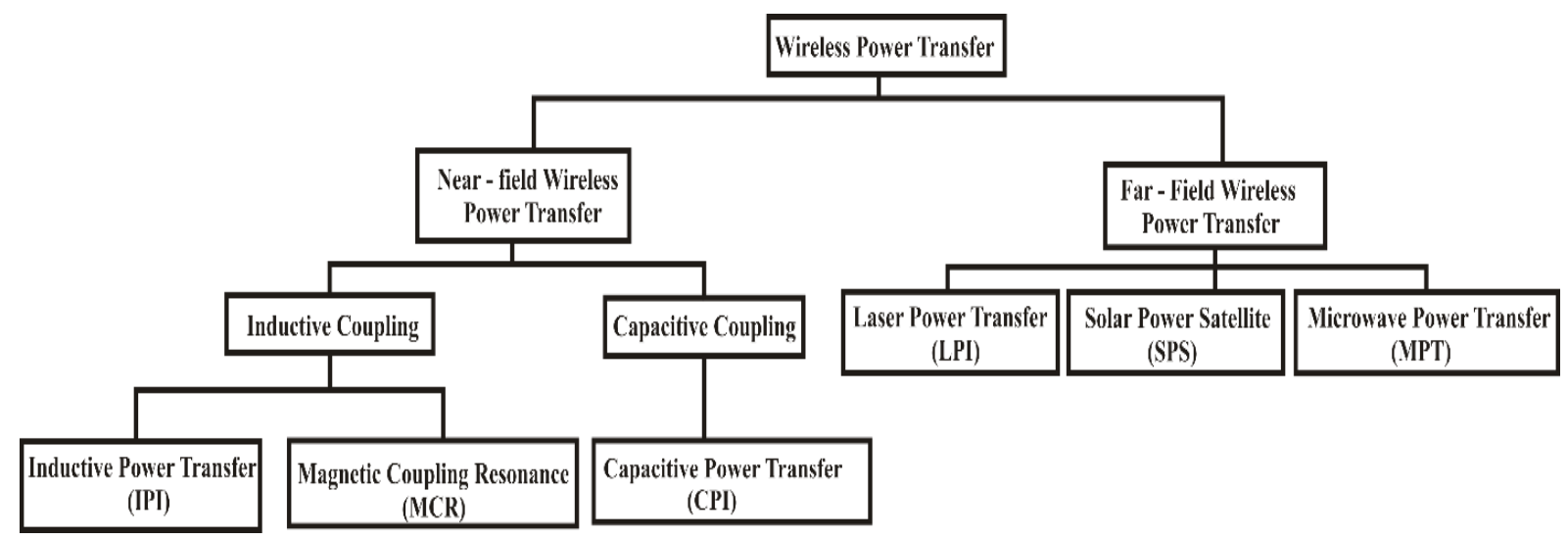

Figure 2: Classification of Wireless Power Transfer

This technology uses the small inductors to transmit power over higher frequencies and also supports a few centimeters maximum charging distance. As a result, portable devices have to be placed quite accurately on the dock to avoid the shortage of a large magnetic field [5]. It is suitable for transferring $5 \mathrm{~W}$ to $10 \mathrm{~W}$ electric current to the load.

The power transfer is established by using digital communications. The power transfer begins by simply placing the device over the charging pad [20]. Owing to its limitation on the charging area, Q $\mathrm{i}$ components can use multiple resonator arrays to create a larger charging area. However, it still does not mitigate the problem and even wastes a lot of power to have individual coils switched on. To keep a strong enough connection, users, therefore, need to align their devices precisely with the magnetic fields [5].

Currently, the wireless charger can get warm during charging and heats the rear part of the device owing to the conductive material it is made up of and the operating frequency. A limited communication protocol is adopted by the Qi to limit the power consumed by multiple coils. With this, the receiving device can tell the charger what proportion power it requires and when it is fully charged. Additionally, the charger can modify its power output to suit any receiving devices and can revert to standby mode on the attainment of full charge or if no device is attached [5].

Companies that utilize this standard for charging their devices are Samsung, LG, Philips, Toyota, Microsoft, and Sony [21]. Qi, a Tightly-Coupled [TC] WPT system is more efficient than the Alliance for Wireless Power (A4WP) Technology. Qi has certain limitations such as single device charging, metal heating, positional alignment necessity, etc. [22].

\subsection{Power Matters Alliance (PMA) Technology}

Another recent technology is the Power Matters Alliance (PMA) [5]. It is a non-profit organization, which develops inductive and resonant power transfer standards. PMA was founded in 2012 to technically harmonize and advance multiple inductive WPT standards, promote WPT within the automobiles industry and popular public infrastructure venues [23]. It uses tightly coupled coils for charging the batteries of mobile devices. PMA transfers power up to 5-10W. The members of PMA are drawn from every known stratum of the mobile device industry, all that constitutes the core value chain [24]. The most prominent members PMA include Duracell, Powermat, LG, Innotek, Panasonic Corporation, Samsung Electronics, Toshiba Corporation, Sony Corporation, Energous Corporation, Freescale Semiconductor Inc, Integrated Device Technology (IDT) and Microsoft Corporation [23].

\section{WIRELESS POWER APPLICATION}

WPT has been used in several fields such as consumer electronics (smartphones, PCs, audio players, tablets, etc.)[30]. Besides the charging of smartphones, cameras, and watches, WPT technologies can also be used for charging TVs [21]. It's also used in medicine, wherein medical implantation applications are used to detect and treat diseases in the human body [2]. WPT also finds its application in charging systems of autonomous underwater vehicles [11], Internet of Things (IoT) [31], electric vehicles [32], wireless sensor network (WSN) [33], unmanned aerial vehicles (UAV) [34]. Highlights of the advantages and disadvantages of wireless power transfer technologies are listed in the following subsections: 


\subsection{Advantages of Wireless Power Transfer}

- It is convenient as cable connection is not needed. [26].

- It is cost-effective.

- It comes in handy as a multi-device charging device.

- Wireless power transfer provides better product durability by delivering reliable power transfer in critical conditions like wet, dirty, and moving environments [27].

- Wireless power transfer also offers the simplicity of product design. Standard features like power ports can be sealed off to the extent that a completely waterproof device is achieved [28]

\subsection{Disadvantages of Wireless Power Transfer}

- Wireless chargers are relatively more expensive at the design stage than a conventional wall socket charger despite their rising popularity [26].

- While using a wireless charger might sound very easy, it is not. To charge a device wirelessly, you have to position the device the right way on the charger. Some chargers even have marked areas and they also direct you the position of the phone. Though this is changing with design upgrades, it's still miles away from being seamless [26].

- Fog, and rain, also affect the practical application of laser and microwave WPT [27].

- With the presence of other communication systems, interference by microwaves occurs, which becomes a disadvantage [27].

\section{COMPARATIVE ANALYSIS OF EXISTING WORK}

In [1], a 3D structured WPT that can transfer power wirelessly in a robotic application was achieved using simulation and experiments at a frequency of 500 $\mathrm{kHz}$. For the winding method that was proposed, a joint made of dual spherical structures was utilized. The joint is made up of a rotating spherical structure inside a huge sphere using a $0^{\circ}-85^{\circ}$ mechanical stud. The transmitter coil ( $\mathrm{Tx}$ ) with a radius of $3.85 \mathrm{~cm}$ was wounded on a spherical structure (with a slot). The mechanical stud can rotate the small sphere up to $45^{\circ}$. The receiver coils (Rx) with a radius of $2.85 \mathrm{~cm}$ have been wounded onto a small sphere structure which produced a displacement angle $a$ in degrees.

The value of $\alpha$ was obtained taking measurements between the joint structures vertical axes. Results showed that at a load (RL) of $20 \Omega$, the hemispherical winding produced an efficiency of up to $96 \%$ at $\alpha=$ $0^{\circ}$. when it was at $85^{\circ}$, the efficiency reduced to less than $10 \%$. For contrast, the optimized WPT under the same load conditions had an efficiency of $95.75 \%$ and $96 \%$ at $\alpha=0^{\circ}$ and $85^{\circ}$ respectively. However, using this proposed method, the magnetic field density is concentrated in the close area around the coils which may be harmful if deployed close to the human body. In addition to this, the authors did not specify the optimization method used for finding the optimal dimensions of the core, coils, and detection circuit elements.

In [37], a professor of Nuclear \& Quantum Engineering at KAIST, and his team build off the ideas of the research team at MIT. Prof Park's team demonstrated great improvement in the distance that electric power can travel wirelessly. The authors developed the "Dipole Coil Resonant System (DCRS)" with a ferrite core wounded in middle for an extended range of inductive power transfer, to a distance of 3, 4 and 5 meters between transmitter and receiver coils with efficiencies of $29 \%, 16 \%$, and $8 \%$ respectively. This team transferred energy based on the oscillating frequency of $20 \mathrm{kHz}$ in the transmitting coil. Measurement for length, width, and height for this system are $3 \mathrm{~m}, 10 \mathrm{~cm}$, and $20 \mathrm{~cm}$ respectively with a low $Q$ factor of 100 . Several experiments were conducted using this system producing results that indicated that operating under $20 \mathrm{kHz}$ produced a maximum power output of $1403 \mathrm{~W}$ at a distance of $3 \mathrm{~m}, 471 \mathrm{~W}$ at $4 \mathrm{~m}$ and $209 \mathrm{~W}$ was obtained at $5 \mathrm{~m}$. $100 \mathrm{~W}$ of electric power transfer gave an overall system power of $36.9 \%$, $18.7 \%$, and $9.2 \%$ for distances of $3 \mathrm{~m}, 4 \mathrm{~m}$, and $5 \mathrm{~m}$ respectively. The Dipole Coil Resonant System is capable of charging up to 40 smartphones once using maximum power output of $209 \mathrm{v}$ at $20 \mathrm{kHz}$. However, it was observed that the coupling was not considered for nonadjacent and adjacent cases [38]. Secondly, the complex structure and large size of the receiver coils make it difficult for them to be integrated into portable consumer devices.

In [39], the authors conducted a study on relay effect via magnetic resonant coupling for wireless power transfer to extend the energy transfer distance. Modeling and numerical analysis were carried out using Computer Simulation Technology (CST) Microwave Studio simulation software. The authors explained that the power efficiency of a typical wireless power transfer system has the poor 
performance to transmit energy at a 1-meter distance which can only reach up to $4.6 \%$ power efficiency. In other to overcome this shortcoming, a relay coil with a diameter of $24 \mathrm{~cm}$, operating at $41.586 \mathrm{MHz}$ frequency was inserted between the transmitter and receiver coils to extend the power transfer distance of the system. After placing the relay coil, the result showed an improvement of up to $50 \%$ increment of power efficiency compared to the system without a relay coil. By adding two relay coils between the Transmitter and receiver, the power efficiency increased up to $75 \%$ at a 1-meter distance of the WPT system. However, the relay coil can sometimes decrease the efficiency when the coupling distance is equal between Tx and Rx coils [40].

In [41], the authors presented analytical and experimental investigations of omnidirectional wireless power transfer using a cubic transmitter, in this paper, the authors proposed the use of cubic transmitter coil to achieve omnidirectional power transfer. The efficiency of the proposed omnidirectional WPT counting on various distances between the transmitter and the receiver along with the transmitter structure was evaluated via analysis and implementation. The result obtained shows that the cubic transmitter was able to achieve an omnidirectional power transfer capability of $60 \%$ efficiency at an operating frequency of $13.56 \mathrm{MHz}$ and an optimum separation of $30 \mathrm{~cm}$. However, the transmitter and receiver coils loop dimension which was set to $20 \mathrm{~cm}$ were too large to be integrated into the consumer devices and cannot be said to be portable as the coil would occupy space in the working area. In addition to this, there was no report of optimization used for finding the optimal dimensions of pick-up coil and detection circuit elements. This could eventually lead to a mismatch between the transmitter and receiver parameters because of the strong relationship between the number of excitation and pick-up coils turns, and the excitation and detection circuits.

In [42], Dual-Band Multi-Receiver Wireless Power Transfer Architecture, Topology, and Control were presented based on a novel reactance steering network (RSN) that can precisely compensate for an arbitrary load reactance by dynamically steering the power between two inverter branches. The transmitter and the receiver are integrated into one WPT system which can operate at dual frequencies. On the transmitter side, the author developed an
RSN based dual-band transmitter that can independently modulate the power transmitted at two frequencies. Instead of merging two independent single frequency transmitters, the RSN architecture reuses the components and creates mutual advantages at two frequencies to improve the performance. At the receiver side, the authors developed a reconfigurable dual-band receiver that can operate at either $100 \mathrm{kHz}$ or $13.56 \mathrm{MHz}$. Multiple dual-band receivers can get up to $30 \mathrm{~W}$ of power from the prototyped RSN transmitter at $74.8 \%$ efficiency at $13.56 \mathrm{MHz}$, and $82.5 \%$ efficiency at 100 $\mathrm{kHz}$ with significant coil misalignment. However, the distance $(2.8 \mathrm{~cm})$ achieved in this paper is too small, a significant coil misalignment of $5 \mathrm{~cm}$ was noticed, the designed transmitting and receiving coils diameter of $10 \mathrm{~cm}$ and $20 \mathrm{~cm}$ were used, which increase the size of the device thereby making the device not portable for commercial use. The design parameters were arbitrarily taken hence, the power attained may not be fully optimized.

In [43], the authors presented optimization design and analysis of the wireless power transfer system with a converter circuit operating at $13.56 \mathrm{MHz}$. A differential evolution (DE) algorithm was used to analyze the model. The WPT system was optimized according to the determined initial parameters of the receiver and transmitter coil with a diameter of $10 \mathrm{~mm}$ and $64 \mathrm{~mm}$. The result obtained showed the power transfer efficiency achieves its maximum value of $30 \%$ when the frequency was $13.56 \mathrm{MHz}$ with an output voltage of $5 \mathrm{~V}$.

In [44], the authors focused on optimizing the output power and transmission efficiency of magnetically coupled resonance (MCR) wireless power transfer system using an existing established mathematical model and Differential Evolution (DE) algorithm. In this paper, optimal parameters such as the distances between the coils, the resonance frequency, and the resistance of the load are obtained employing a differential evolution algorithm to optimize the output power and transmission efficiency of the twocoil system and four-coil MCR WPT. The output power and transmission efficiency result obtained for two coils with coil radius $0.1 \mathrm{~m}$ at an optimized transmission distance of $0.2 \mathrm{~m}$ were showed to be $0.46 \mathrm{~W}$ and the result for four coils at $0.3 \mathrm{~m}$ distance was shown to be $10 \mathrm{~W}$ for a given resonance frequency of $1 \mathrm{MHz}$ respectively. By comparing and analyzing simulation results, the output power and transmission efficiency of the MCR WPT system with 
designated coil structure was improved. Though the differential evolution optimization method used in this research papers was simple to implement, however, the model requires substantially longer simulation time and the probability of getting the nearest optimal point is very less. Premature convergence is the most frequent happening in $\mathrm{DE}$. In [45], the authors proposed the use of a novel Particle Swarm Optimization (PSO) to address the problem of low transfer efficiency in a magneticallycoupled resonant wireless power transfer (MCRWPT) system. The analytical calculation, numerical simulation, and experiments were also conducted in this paper. The searching for optimal frequency in the MCR-WPT systems with or without frequency splitting was simulated and finally, the theoretical analysis was experimentally validated. The result shows that at transfer distance of $14 \mathrm{~cm}$, with a resonant frequency of $8 \mathrm{MHz}$, and a coil radius of $15 \mathrm{~cm}$, the peak of voltage across load drops sharply from $12 \mathrm{~V}$ to $4.2 \mathrm{~V}$ as the transfer distance decreases from $14 \mathrm{~cm}$ to $8 \mathrm{~cm}$. The voltage remains at a higher value in the MCR-WPT system using the proposed PSO algorithm when transfer distance changes. However, the proposed PSO algorithms have the drawback of maintaining a lot of complex algorithmic control parameters to achieve the better search path in determining, it also has the drawback of untimely convergence and it also drops into regional optimum. The authors in [46] focused on the optimization of a wireless power transmission system using the Simulated Annealing (SA) optimization algorithm to determine the parameters of the parasitic wires. To optimize the efficiency of wireless power transmission, the authors applied Simulated Annealing (SA) optimization algorithm to determine the parameters of the parasitic wires by carefully adjusting the position, size, and geometry of the parasitic wires. The result obtained shows an increase in the overall efficiency of the system by $30 \%$ at $1 \mathrm{GHz}$ frequency, parasitic wire radius of 0.02 $\lambda$, and distance of $0.42 \lambda$ in contrast to the existing design of the wireless power transfer system. Though SA can avoid being trapped in local minima, however, the SA method adopted in the design cannot show whether it has found an optimal solution. Some other method (e.g. branch and bound) is required to do this; also this method requires infinite time as there is no provision to specify the maximum number of iterations. Since the research work required to account for different constraints, to fine-tune the parameters of the algorithm can be rather delicate.

The authors in [48] investigated a 3-coil MCR WPT system based on the equivalent circuit and fundamental harmonic analysis (FHA) method to improved transmission distance and also to achieve a stable power transmission of a wireless transfer system. The result obtained shows that by placing the repeater coil at the center of the sending and receiving coil, the stable output power of about $70 \mathrm{~W}$ can be achieved at a frequency of $200 \mathrm{kHz}$ and a coil radius of $11 \mathrm{~cm}$. However, additional coil increases the complexity of the system and it also implies that more turning work will be done to get all the parameters aligned, thereby making it difficult to maintain optimal performance in a harsh environment for the practical applications [49].

The authors in [50] proposed an impedance matching approach based on coupling tuning using the Advanced Design System (ADS) simulator to improve and maximize the transfer efficiency of 4coils resonant coupled (the diameter of each loop being $20 \mathrm{~cm}$ )WPT system at $12 \mathrm{~Hz}$ operating frequency. The maximum efficiency of the system was calculated as a function of the loaded quality factors and coupling coefficients. The effect of coil distance variation is studied through simulation and the results showed improved system performance. However, the impedance matching circuit consists of many passive components such as inductors, capacitors, and switches that occupied a large area and increase the system loss.

\section{CONCLUSION}

Based on the literature reviewed in this paper, the selected researchers have developed and experimented with different compensation techniques for different applications to optimize the performance of the wireless power transfer system using various optimization techniques. Despite the extensive work done by researchers in the field of wireless power transfer, WPT's full potential has not been realized up till now in term of transfer power and distance, frequency and coils dimensions as the current designed transmitter and receiver coils are too large to be integrated into the consumer devices and cannot be said to be portable as the coil would occupy space in the working area. Hence the need for the continuation of research in this field as there are many promising applications suited for wireless power transfer. 
Table 1. Comparative analysis of existing work based on optimization and topology used

\begin{tabular}{|c|c|c|c|c|c|c|c|c|}
\hline $\begin{array}{l}\text { Ref } \\
\text { no }\end{array}$ & Year & $\begin{array}{l}\text { Optimization/simulation } \\
\text { method used }\end{array}$ & $\begin{array}{l}\text { Topology } \\
\text { method } \\
\text { used }\end{array}$ & Frequency & $\begin{array}{l}\text { Coil } \\
\text { diameter }\end{array}$ & $\begin{array}{l}\text { Vout/Output } \\
\text { power }\end{array}$ & Distance & $\begin{array}{l}\text { Efficiency } \\
\%\end{array}$ \\
\hline 1 & 2018 & ANSYS electronics & $\begin{array}{l}\text { Series- } \\
\text { series }\end{array}$ & $500(\mathrm{kHz})$ & $\begin{array}{l}\text { Tx coil } \\
=7.7 \mathrm{~cm} \\
\text { Rx coil } \\
=5.7 \mathrm{~cm}\end{array}$ & $5 \mathrm{v}$ & & 96 \\
\hline 37 & 2014 & Numerically modeled & & $20(\mathrm{kHz})$ & & $\begin{array}{l}\text { 1,403W, } \\
471 W \\
209 W\end{array}$ & $\begin{array}{l}3 m \\
4 m \\
5 m\end{array}$ & $\begin{array}{l}29 \\
16 \\
8\end{array}$ \\
\hline 39 & 2016 & $\begin{array}{l}\text { Computer Simulation } \\
\text { Technology (CST) } \\
\text { Microwave Studio } \\
\text { simulation software }\end{array}$ & $\begin{array}{l}\text { relay } \\
\text { resonator } \\
\text { coil }\end{array}$ & $\begin{array}{l}\text { 41.587(MHz) } \\
29.982(\mathrm{MHz})\end{array}$ & $\begin{array}{l}24 \mathrm{~cm} \\
28 \mathrm{~cm}\end{array}$ & & $1 \mathrm{~m}$ & $\begin{array}{l}71 \\
89\end{array}$ \\
\hline 41 & 2018 & Mathematical analysis & & $13.56(\mathrm{MHz})$ & & & $30 \mathrm{~cm}$ & 60 \\
\hline 42 & 2019 & & $\begin{array}{l}\text { reactance } \\
\text { steering } \\
\text { network } \\
\text { (RSN) }\end{array}$ & $\begin{array}{l}100(\mathrm{kHz}) \\
13.56(\mathrm{MHz})\end{array}$ & $\begin{array}{l}10 \mathrm{~cm} \\
20 \mathrm{~cm}\end{array}$ & $\begin{array}{l}15 \mathrm{~W} \\
10 \mathrm{~W}\end{array}$ & $2.8 \mathrm{~cm}$ & $\begin{array}{l}80 \\
70\end{array}$ \\
\hline 43 & 2017 & $\begin{array}{l}\text { differential evolution (DE) } \\
\text { algorithm }\end{array}$ & & $13.56(\mathrm{MHz})$ & $\begin{array}{l}T x=64 \mathrm{~mm} \\
R x=10 \mathrm{~mm}\end{array}$ & $5 \mathrm{~V}$ & & 30 \\
\hline 44 & 2018 & $\begin{array}{l}\text { mathematical model and } \\
\text { Differential Evolution (DE) } \\
\text { algorithm }\end{array}$ & & $1(\mathrm{MHz})$ & $0.2 \mathrm{~m}$ & $\begin{array}{l}0.46 \mathrm{~W} \\
0.10 \mathrm{~W}\end{array}$ & $\begin{array}{l}0.2 \mathrm{~m} \\
0.3 \mathrm{~m}\end{array}$ & $\begin{array}{l}0.93 \\
0.67\end{array}$ \\
\hline 45 & 2018 & $\begin{array}{l}\text { Particle Swarm } \\
\text { Optimization (PSO) } \\
\text { algorithm }\end{array}$ & & $8(\mathrm{MHz})$ & $30 \mathrm{~cm}$ & $4.2 \mathrm{~V}$ & $14 \mathrm{~cm}$ & 85 \\
\hline 46 & 2016 & Simulated Annealing (SA) & & $1(\mathrm{GHz})$ & $0.04 \lambda$ & & $0.42 \lambda$ & 30 \\
\hline 48 & 2017 & $\begin{array}{l}\text { fundamental harmonic } \\
\text { analysis (FHA) }\end{array}$ & & $200(\mathrm{kHz})$ & $22 \mathrm{~cm}$ & $70 \mathrm{~W}$ & & \\
\hline 50 & 2016 & $\begin{array}{l}\text { Advanced Design System } \\
\text { (ADS) simulator }\end{array}$ & & $12 \mathrm{~Hz}$ & $20 \mathrm{~cm}$ & & & \\
\hline
\end{tabular}

\section{REFERENCES}

[1] M. A. Houran, X. Yang, and W. Chen, "Magnetically Coupled Resonance WPT: Review of Compensation Topologies, Resonator Structures with Misalignment, and EMI Diagnostics" , MDPI Journal, 2018.

[2] A. M. Jawad, R. Nordin, and S. K. Gharghan, "Opportunities and Challenges for Near-Field Wireless Power Transfer: A Review", MDPI Journal , 2017, pp. 1-28.

[3] B. Zhu, J. Li, W. Hu, and X. Gao, "Review of Magnetic Coupling Resonance Wireless Energy Transmission", International Journal of u- and e-Service, Science and Technology vol. 8, no. 3, pp. 257-272, 2015.

[4] K. Arai, "Wireless power transfer solution for 'Things' in the internent of THings", proceedings of the Future Technologies Conference (FTC), vol. 1. 2018.

[5] M. Shidujaman, H. Samani, and M. Arif,
"Wireless power transmission trends," 2014 Int. Conf. Informatics, Electron. Vision, ICIEV 2014, 2014.

[6] R. Zhang, "Communications and Signals Design for Wireless Power Transmission," Cs.IT, 2016.

[7] H. Hong, D. Yang, and S. Won, "The Analysis for Selecting Compensating Capacitances of Two-Coil Resonant Wireless Power Transfer System," Proc. - 1st IEEE Int. Conf. Energy Internet, ICEI 2017, pp. 220-225, 2017.

[8] Y. Zhang, Z. Yan, Z. Liang, S. Li, and C. Mi, "An LCL-N Compensated Strongly-Coupled Wireless Power Transfer System for HighPower Applications," 2019 IEEE Appl. Power Electron. Conf. Expo., pp. 3088-3091, 2019.

[9] P. S. Patil and S. K. Padaganur, "Challenging Issues In Wire Less Power Transmission Methods A Survey," International Journal of Scientific and Technology Research vol. 7, no. 8, pp. 136-139, 2018. 
[10] A. Massa, G. Oliveri, F. Viani, and P. Rocca, "Array designs for long-distance wireless power transmission: State-of-the-art and innovative solutions," Proc. IEEE, vol. 101, no. 6, pp. 1464-1481, 2013.

[11] X. Lu, P. Wang, D. Niyato, D. I. Kim, Z. Han, and C. Engineering, "Wireless Charging Technologies: Fundamentals, Standards, and Network Applications," EEE Communications Surveys and Tutorials, 2016, pp. 1-40.

[12] C. Mkasanga, "A Feasibility Study of Wireless Charging"

https://www.academia.edu/9567045/A Feasi bility Study of Wireless Charging 2012.

[13] A. Arshad, S. Khan, A. H. M. Zahirul Alam, and R. Tasnim, "Investigation of inductive coupling approach for non-contact bidirectional transfer of power and signal," 2012 Int. Conf. Comput. Commun. Eng. ICCCE 2012, no. July, pp. 570573, 2012.

[14] V. Shevchenko and S. Member, "Compensation Topologies in IPT Systems: Standards, Requirements, Classification, Analysis, Comparison and Application," IEEE Access , pp. 120559-120580, 2019.

[15] Y. Luo, Y. Yang, S. Chen, and X. Wen, "Power Waves-based Analysis of Magnetically Coupled Resonant Wireless Power Transfer", IEEE Accss, pp. 878-882, 2017.

[16] Sang Wook Han, "Wireless Interconnect using Inductive Coupling in 3D-ICs https://deepblue.lib.umich.edu/bitstream/han dle/2027.42/94034/swhanpns 1.pdf?sequenc $\mathrm{e}=12012$.

[17] X. Chen and X. Gong, "Modeling of wireless power system with giant magnetostrictive material load under multi-field coupling," Conf. Proc. - IEEE Appl. Power Electron. Conf. Expo. - APEC, no. Mci, pp. 3100-3105, 2017.

[18] P. Bakolia, "Wireless Power Transmission - The Future of Power Transmission System. By" , Journal of Advanced Computing and Communication Technologies, no. 5, pp. 4854, 2017.

[19] E. Waffenschmidt, "Wireless Power for Mobile Devices.", NTELEC, International Telecommunications Energy Conference (Proceedings), 2011

[20] T. S. C. Rao, "Categories, Standards and Recent Trends in Wireless Power Transfer: A Survey" Indian Journal of Science and Technology, vol. 9, no. May 2016.

[21] X. Mou, S. Member, and H. Sun, "Wireless
Power Transfer: Survey and Roadmap", IEEE Vehicular Technology Conference, no. 646470, pp. 1-13, 2015

[22] P. Dubal, "Rezence - Wireless Charging Standard based on Magnetic Resonance," Int. J. Adv. Res. Comput. Commun. Eng., vol. 4, no. 12, pp. 198-200, 2015.

[23] Vladislav Khayrudinov " Wireless Power Transfer system : Development and Implementation", ResearchGate, 2015

[24] S. H S, "Wireless Charging Techniques - A Survey," Int. J. Res. Appl. Sci. Eng. Technol., vol. V, no. III, pp. 480-484, 2017.

[25] V. Khayrudinov, "Wireless Power Transfer system: Development and Implementation", ResearchGate, 2015

[26] R. Gupta, "Is Qi Wireless Charging Worth It: Pros and Cons," https://www.guidingtech.com/qi-wirelesscharging-pros-cons/ pp. 1-7, 2018.

[27] A. V. Kumar, P. Niklesh, and T. Naveen, "Wireless Power Transmission," International Journal of Engineering and Application, vol. 1, no. 4, pp. 1506-1510.

[28] W. Is and W. Power, "Introduction to Wireless Power Transfer: Benefits, Markets, and What is your opinion ?", Mendeley, pp. 1-2, 2019.

[29] S. Das, "Review Paper on Wireless Power Transmission for Charging Mobile Devices,", mendeley, vol. 6, no. 3, pp. 20751-20755, 2017.

[30] M. Fareq, M. Fitra, M. Irwanto, S. Hasan, and M. Arinal, "Low wireless power transfer using Inductive Coupling for a mobile phone charger" IOPscience, vol. 495, pp. 1-7, 2014.

[31] A. Uchida, "Three-dimensional Wireless Power Transfer Method to Realize Efficient Charging of IoT Devices" FUJITSU Sci. Tech. J., Vol. 53, No. 2, pp. 51-56, pp. 51-56 February 2017

[32] N. W. P. Transfer, "Wireless Charging of Electric Vehicles" https://www.ncbi.nlm.nih.gov/books/NBK481 644/\#, no. Dc, pp. 1-6, 2019.

[33] R. A. Assistant, A. M. Assistant, and R. K. Student, "Various Optimization techniques used in Wireless Sensor Networks Key Words:" International Research Journal of Engineering and Technology (IRJET), pp. 2085-2090, 2016.

[34] M. Simic, C. Bil, and V. Vojisavljevic, "Investigation in wireless power transmission for UAV charging," Procedia - Procedia 
Comput. Sci., vol. 60, pp. 1846-1855, 2015.

[35] I. Control, "Power Transfer System Application," https://www.google.ch/patents/US201202993 92, pp. 255-259, 2017.

[36] S. Li, W. Li, S. Member, J. Deng, and S. Member, "A Double-Sided LCC Compensation Network and Its Tuning Method for Wireless Power Transfer", IEEE Transactions on Vehicular Technology vol. 64, no. 6, pp. 22612273, 2015.

[37] C. Park et al., "Innovative 5m-off-distance Inductive Power Transfer Systems with Optimally Shaped Dipole Coils," IEEE Transactions on Power Electronics, vol. 8993, no. c, 2014.

[38] C. Saha et al., "Wireless power transfer using relay resonators Wireless power transfer using relay resonators," AIP Conference Proceedings, vol. 263902, 2018.

[39] N. A. Rashid et al., "Design and Optimization of Magnetic Resonance Coupling by using Relay Effect.", 2016 10th European Conference on Antennas and Propagation, EuCAP, 2016

[40] N. A. Rashid, M. N. M. Yasin, K. Kamardin, N. Ramli, M. Jusoh, and S. A. Z. Murad, "A study on Relay Effect via Magnetic Resonant Coupling for Wireless Power Transfer, " MATEC Web of Conferences, vol. 01095, 2016.

[41] N. Ha-van, "Analytical and Experimental Investigations of Omnidirectional Wireless Power Transfer Using a Cubic Transmitter", IEEE Transactions on Industrial Electronics, vol. 65, no. 2, pp. 1358-1366, 2018.

[42] M. Liu and M. Chen, "Dual-Band Multi-Receiver Wireless Power Transfer: Architecture, Topology, and Control" , Conference Proceedings - IEEE Applied Power Electronics Conference and Exposition - APEC, 2019

[43] X. Zhang, X. Zhang, C. Zhang, S. Yao, H. Qi, and $\mathrm{Y}$. Xu, "Optimal design and analysis of wireless power transfer system with converter circuit", EURASIP Journal on Wireless Communications and Networking pp. 1-6, 2017.
[44] A. You, M. A. Y. Be, and I. In, "Optimization of output power and transmission efficiency of magnetically coupled resonance wireless power transfer system", AIP Advances, vol. 056625, 2018.

[45] M. Wang, J. Feng, Y. Shi, M. Shen, and J. Jing, "A Novel PSO-Based Transfer Efficiency Optimization Algorithm for Wireless Power Transfer", Progress In Electromagnetics Research, vol. 85, no. July, pp. 63-75, 2018.

[46] A. R. C. Cheah, K. H. Yeap, K. Hirasawa, K. C. Yeong, and $\mathrm{H}$. Nisar, "Optimization of a Wireless Power Transmission System" Proceedings of the International MultiConference of Engineers and Computer Scientists, vol. II, pp. 16-18, 2016.

[47] F. Zhu, Y. Tang, P. Cao, and H. Ma, "A Method of Resonant Frequency Optimized Selection for A SP Inductive Coupled Power Transfer System" IEEE Access, vol. 3, pp. 3-7, 2017.

[48] W. Ye, L. Chen, F. Liu, S. Member, X. Chen, and X. Wang, "Analysis and Optimization of 3Coil Magnetically Coupled Resonant Wireless Power Transfer System for Stable Power Transmission", IEEE Access, pp. 2584-2589, 2017.

[49] H. Zhou, B. Zhu, W. Hu, Z. Liu, and X. Gao, "Modelling and Practical Implementation of 2Coil Wireless Power Transfer Systems" Journal of Electrical and Computer Engineering, Volume 2014, Article ID 906537, 2014.

[50] S. Das Barman, A. W. Reza, and N. Kumar, "Coupling Tuning Based Impedance Matching for Maximum Wireless Power Transfer Efficiency" Journal of Computer Science \& computational Mathematics, vol. 6, no. 4, 2016.

[51] S. Ansari, A. Das, and A. Bhattacharya, "TwoCoil System with class-E Resonant HighFrequency Inverter" IEEE International Conference on Signal Processing and Integrated Networks pp. 269-273, 2019.

[52] S. Lee, K. Yi, and M. Kim, "Distance Using a GaN-Based Full-Bridge Inverter" MDPI Journal, 2019. 